\title{
Heat Transfer Characteristics of a Pharyngeal Cooling Cuff for the Treatment of Brain Hypothermia*
}

\author{
Koji FUMOTO $^{* *}$, Yoshimasa TAKEDA ${ }^{* * *}$, Hiroshi HASHIMOTO ${ }^{* * * *}$, \\ Masatomo KOKUBU $^{* * * *}$ and Tsuyoshi KAWANAMI ${ }^{* * * * *}$ \\ ${ }^{* *}$ Department of Mechanical Engineering, Kushiro National College of Technology, \\ Otanoshike-Nishi 2-32-1, Kushiro, Hokkaido 084-0916, Japan \\ E-mail: fumoto@mech.kushiro-ct.ac.jp \\ ${ }^{* * *}$ Department of Anesthesiology and Resuscitology, Graduate School of Medicine, Dentistry, and \\ Pharmaceutical Sciences, Okayama University, \\ 2-5-1, Shikada-cho, Okayama 700-8558, Japan

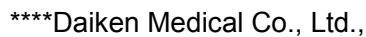 \\ 2-6-2, Ayumino, Izumi, Osaka 594-1157, Japan \\ *****Division of Mechanical Engineering, Graduate School of Engineering, Kobe University, \\ 1-1, Rokkodai-cho, Nada-ku, Kobe, Hyougo 657-8501, Japan
}

\begin{abstract}
In the present study, the characteristics of both flow and heat transfer in a pharyngeal cooling cuff for the treatment of brain hypothermia were investigated experimentally and numerically. The pharyngeal cooling cuff, which is a balloon-like structure placed in the pharynx, was developed for medical purposes. As a method for controlling the brain temperature, cooling water, which is physiological saline at $5^{\circ} \mathrm{C}$, is injected into the cuff in order to cool the common carotid artery, which is adjacent to the pharynx. In this study, the heat transfer characteristics between the cuff wall and phantom body, which was considered to be equivalent to the human body, were experimentally determined, and the flow behaviour in the cuff was observed in detail. Furthermore, a three-dimensional numerical simulation was carried out to investigate both the flow velocity and temperature distribution in the cuff.
\end{abstract}

Key words: Pharyngeal Cooling, Brain Hypothermia, Medical Engineering, Numerical Simulation, Heat Transfer

\section{Introduction}

In Japan, cerebropathy, including cerebrovascular disorder, is one of the causes of death following malignant neoplasms and heart diseases ${ }^{(1)}$. Crisis prediction in the case of cerebrovascular disorders and elucidation of the mechanism underlying the development of these disorders are the need of the day. Further, the establishment of a good method for treating the recurrence of neurological condition, including the society return, is strongly desired. Hayashi ${ }^{(2) \sim(3)}$ revealed that one of the therapies for lowering the brain temperature in the case of serious wound injuries to the head, severe cerebrovascular disorders, or brain ischemia was cardiopulmonary stop; Patients treated with this method were found to have a good neurological prognosis ${ }^{(4) \sim(5)}$. At present, low-temperature brain therapy enables the controls of the brain temperature and intracranial pressure in the case of patients with severe brain damage who satisfy the condition variously. This therapy has been introduced to repair nerve cells damaged during the initial brain damage, and to prevent the development of secondary brain damage. Since the release of neurotransmitters and action of free radicals are both drastically suppressed at brain temperatures of $32^{\circ} \mathrm{C}$ or less, it is said that 
the brain is protected against damage. For the purpose of treatment, brain hypothermia is divided into mild hypothermia (core temperature, $\left.34^{\circ} \mathrm{C}\right)$ and moderate hypothermia $\left(32^{\circ} \mathrm{C}\right)$. The temperature of the brain is lowered and maintained at low temperatures by lowering the body temperature. The latter is achieved by covering the whole body with a water-cooled blanket. In this method, the patient's body is wrapped with a water-cooled blanket, and cold water (approximately $20^{\circ} \mathrm{C}$ ) is circulated through the blanket. It is also possible to simultaneously maintain the brain temperature at the desired degree and strictly monitor the temperature. However, this management strategy, i.e., conventional hypothermia treatment, requires a great deal of time to bring about changes in body temperature and for acute state transition of the pathology. Therefore, research and development on medical instruments such as water-cooling blankets, headgear, and mufflers for the purpose of cooling of body parts and lowering the brain temperature are widely being conducted ${ }^{(6)}$.

Takeda et al. ${ }^{(7) \sim(8)}$ developed the pharyngeal cooling cuff by analyzing the anatomy of the pharynx division. This cuff is inserted through the oral cavity of the patient, and it can cool the common carotid artery, which is located approximately $10 \mathrm{~mm}$ outside the pharynx division. Thus, cooling and temperature control of the brain is possible via the cooling of the main blood vessel that supplies the brain. In comparison with conventional whole-body cooling, the pharyngeal cuff method rapidly lowers the brain temperature. Moreover, the countermeasures of acute brain temperature management and early brain cooling during life-threatening emergencies related to brain diseases are possible with the latter method. For acute phase of brain cooling, we assume that physiological saline at $5^{\circ} \mathrm{C}$ should be used to cool the cuff. During animal testing ${ }^{(9)}$, this cuff has been shown to produce a good cooling effect on the brain. However, the optimum cooling efficiency of this cuff has not been determined since the structure of this device is based on the pharynx shape.

On the basis of this background, we aimed to understand the flow and heat transfer characteristics of the pharyngeal cooling cuff. In this report, in order to evaluate pharyngeal cooling, we analyzed the heat transfer between the cuff and a phantom body (hereafter referred to as phantom), which was considered to be equivalent to the human body. In particular, we focused on the thermal transport characteristics within the cuff. Further, computational fluid dynamics (CFD) analysis was carried out to analyze the flow in the cuff, and the cooling efficiency was examined under various conditions. These results are useful as they provide the basic information required to develop the clinical applications of the cuff and to improve the cuff to ensure better cooling of the pharynx. Future studies will address transport within the surrounding tissue and the effectiveness of carotid artery blood cooling.

\section{Nomenclature}

$\begin{array}{ll}\mathrm{D} & : \text { Diameter } \\ \mathrm{L} & : \text { Length } \\ \mathrm{Q} & : \text { Heat quantity } \\ \mathrm{q} & : \text { Heat flux } \\ \mathrm{S} & : \text { Surface area } \\ \mathrm{T} & : \text { Temperature } \\ \Delta \mathrm{T} & : \text { Temperature difference } \mathrm{t} \\ \mathrm{U} & : \text { Velocity } \\ \mathrm{V} & : \text { Inlet flow } \\ \mathrm{X} & : \text { Local position } \\ \eta & : \text { Cooling efficiency } \\ \rho & : \text { Density }\end{array}$




Subscript
$1 \sim 6 \quad$ Thermocouple
in $\quad:$ Inflow
$\mathrm{L} \quad:$ Liquid phase
out $\quad$ Outflow
Pha : Phantom

\section{Experimental setup and procedures}

\subsection{Pharyngeal cooling cuff}

The structure of the pharyngeal cooling cuff and a schematic diagram of the working of the cooling cuff are shown in Figures 1 and 2, respectively. In the case of patients with difficulties in voluntary respiration, the pharyngeal cooling cuff is inserted into the pharynx division along with the tube for artificial respiration. Therefore, the shape of the cuff is based on the shape of the pharynx division. The upper and lower parts of the cuff are placed in the pharynx and upper esophageal divisions, respectively. The cooling cuff, which is a balloon-like structure, is $0.5 \mathrm{~mm}$ in thickness and made of polyvinyl. It is $250 \mathrm{~mm}$ long with a total volume of $100 \mathrm{ml}$. The cooling cuff has an inlet tube and 2 outlet tubes, which are 4 $\mathrm{mm}$ in diameter. The basic flow behavior within the cooling cuff is as follows. Cooling water, which is physiological saline at around $5^{\circ} \mathrm{C}$, flows into the inlet tube. The cooling water passes through the cuff and reaches the tip of the cuff. At the tip, the inlet

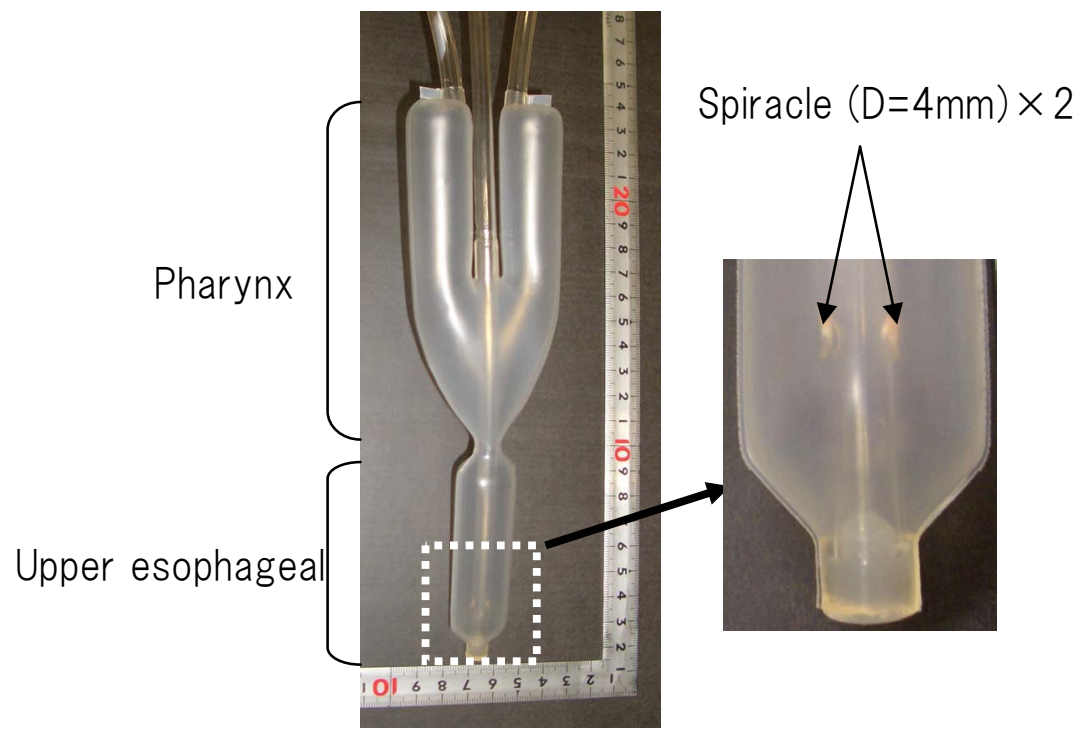

Figure 1 Appearance of pharyngeal cooling cuff

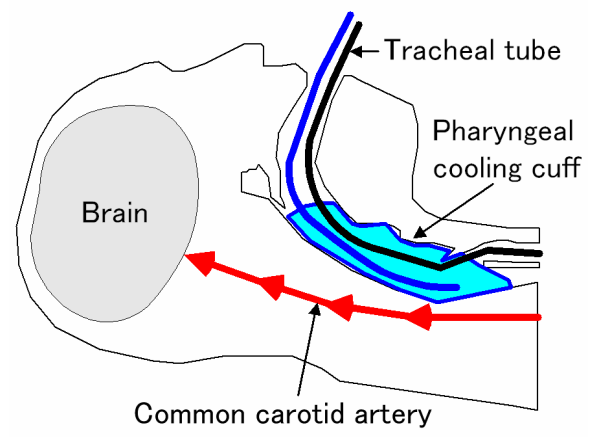

Figure 2 Image of the pharyngeal cooling cuff for brain hypothermia treatment 
tube has 2 spiracles, $4 \mathrm{~mm}$ in diameter, on the tube wall. After passing through the spiracles, the cooling water, which is between the cuff and inlet tube, flows to the upper part of the cuff. During this time, the cuff wall is cooled by heat transfer between the inner cuff wall and the cooling water. Finally, in the upper part of the cuff, the cooling water flows in 2 directions and out through the outlet tubes.

\subsection{Experimental apparatus and procedures}

A schematic diagram of the experimental facility used in the present investigation is shown in Figure 3. The experimental apparatus includes a heater; a cooling water circulatory system, including a flow-control device; measuring equipment; and the cuff that is placed in the phantom. The cooling water circulation apparatus can adjust the temperature and quantity of cooling water flowing into the cuff. Distilled water was used as the working fluid. The running water temperature was measured with a K-type thermocouple $(\mathrm{D}=0.3$ $\mathrm{mm}$ ) inserted into each tube. The phantom can be maintained at temperatures ranging from room temperature to $45^{\circ} \mathrm{C}$, by adjusting the heater. The K-type thermocouple $(\mathrm{D}=0.3 \mathrm{~mm})$ was positioned in the phantom at 6 points $\left(\mathrm{T}_{1} \sim \mathrm{T}_{6}\right.$; positions are shown in Figure 7) between 2 places, cuff and phantom, and the phantom temperature and local surface temperature of the cuff were measured. All thermal data were fed into computers through a data logger at $1 \mathrm{~s}$ intervals.

The phantom was maintained at the setting temperature, after the specified quantity of cold water at the desired temperature was passed through the cuff, while the output of the band heater, which was wound around the phantom circumference, was adjusted by heat transfer. In the temperature setting of the phantom, the mean values of the 2 thermocouples installed near the cuff were adjusted to the phantom temperature. The data were collected, after a steady state was achieved.

For the visualization experiment, the cuff was placed in the standing position after it was taken out of the phantom, and the observations were made. The water was mixed with insoluble fluorescent particles $\left(d=165 \mu \mathrm{m}, \rho=1050 \mathrm{~kg} / \mathrm{m}^{3}\right.$; to trace the water dispersion) for better visualization. A specified quantity of this water was supplied, and the flow behavior was recorded with a digital video camera.

The inflow temperature ranged from $\mathrm{T}_{\mathrm{L}}=3 \sim 10^{\circ} \mathrm{C}$ during the experimental conditions, and the inflow, $\mathrm{V}=100 \sim 1500 \mathrm{ml} / \mathrm{min}$. The temperature of the phantom was maintained at

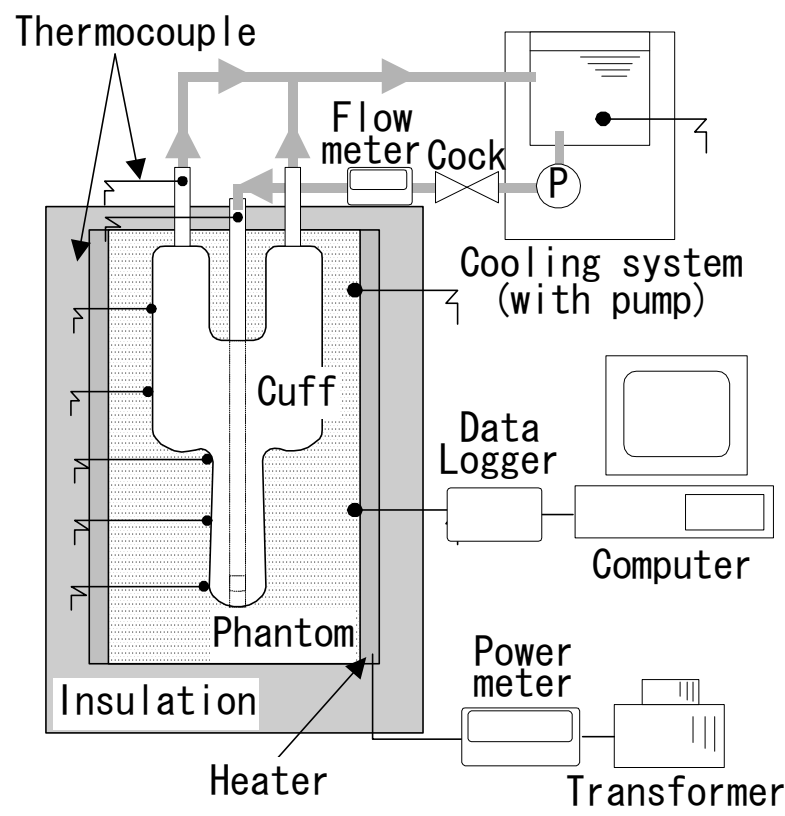

Figure 3 Experimental apparatus 
$\mathrm{T}_{\text {Pha }}=35 \sim 38^{\circ} \mathrm{C}$. In addition, the measurement accuracy of this experiment was as follows. The relative temperature difference of the thermocouple was within $0.4 \mathrm{~K}$. The temperature reading error was within $\pm 2.5 \%$, considering the accuracy of the measuring equipment. The erratic readings of the inflow were within $\pm 0.02 \%$ of the accuracy of the flow meter.

\subsection{Human body equivalent phantom}

The phantom used in this experiment was a wet type one constructed with agar, boric acid, and water. The mould was removed, and the phantom was left to solidify and form a cylindrical vessel. This vessel was used for the experiment and placed in an independent (standing) position. The proportion of the components used to construct of the phantom is shown in Table 1. Furthermore, the thermal conductivity of the phantom measured by the unsteady probe technique as well as the thermal conductivity of the representative organism is shown in Table $2^{(10)}$. In the experiment, the phantom was lap coated to prevent changes in the water content due to evaporation from the exterior.

Table 1 Principal components of the phantom body

\begin{tabular}{|c|c|c|c|}
\hline Component & Agar & Boric acid & Water \\
\hline Ratio $\left(\mathrm{wt}^{\mathrm{o}} \mathrm{)}\right)$ & 3.0 & 1.2 & 95.8 \\
\hline
\end{tabular}

Table 2 Thermal conductivity

\begin{tabular}{|c|c|c|}
\hline & Phantom & Ref. (10) \\
\hline $\begin{array}{c}\text { Thermal conductivity } \\
(\mathrm{W} /(\mathrm{mK}))\end{array}$ & 0.532 & $0.34 \sim 0.68$ \\
\hline
\end{tabular}

\subsection{Calculation grid and numerical analysis method.}

The GAMBIT grid generation software was used for analysis of three-dimensional $\mathrm{CAD}$ data from the cuff to prepare a calculation grid. The calculation grid comprised a triangular 8-faced cone grid in the cuff interior and in the vicinity of the wall. The calculation grid is shown in Figure 4 (left: coronal plane, right: median section). The calculation grid number of the cuff is shown in Table 3 . In the numerical analysis, the thermo-hydrodynamic analysis software FLUENT was used.

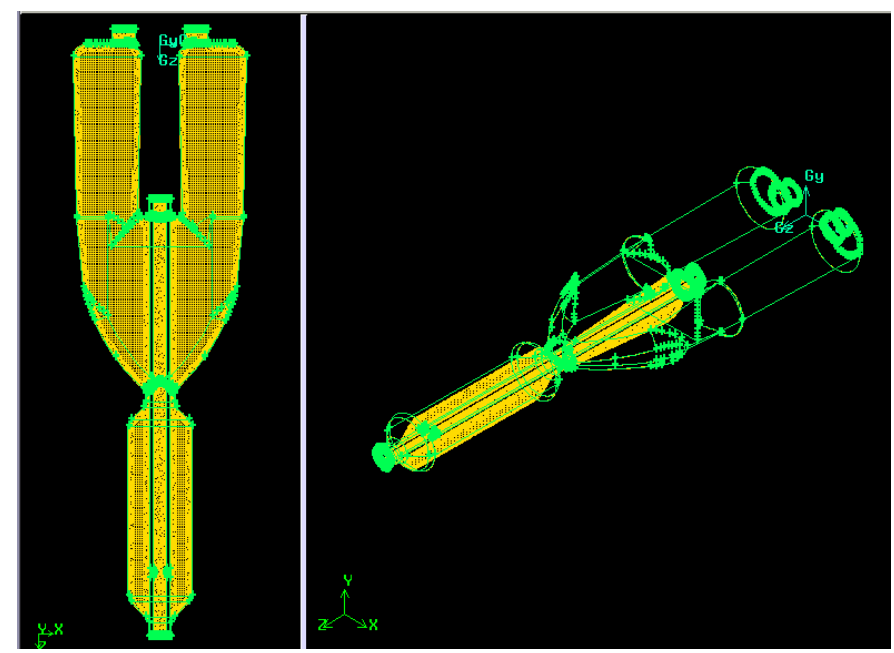

Figure 4 Numerical grid for the cuff (3D) 
Table 3 Grid size of the cuff model

\begin{tabular}{|c|c|c|}
\hline Cells & Faces & Nodes \\
\hline 803,759 & $1,805,366$ & 261,420 \\
\hline
\end{tabular}

\section{Results and Discussion}

\subsection{Surface temperature of cuff}

The relationship between surface temperature and inflow at different positions in the cuff is shown in Figure 5. The phantom temperature and inflow temperature were fixed at $37^{\circ} \mathrm{C}$ and $5^{\circ} \mathrm{C}$, respectively. The horizontal axis of the figure indicates the thermometry position of the cuff. When the inflow is small, the cuff surface temperature is generally higher. Furthermore, no large differences in the inflow temperature were observed at the different cuff positions. However, the surface temperatures at positions 3, 5 and 6 were lower than the temperature at the other positions in the cuff. Due to these reasons, another factor was considered. At around position 3, there was an increase in the flow velocity with a rapid decrease in the water course at the cross section of the cuff center, which led to an increase in the heat transfer coefficient, thereby enhancing the cooling effect. Furthermore, near position 5, after the fluid that passed through the center of the cuff was pooled in the bifurcation, this fluid was divided between both the cuff branches; the water pools again, and the local heat transfer increases. Thus, there is increased heat transfer at position 6 due to this complicated flow, including the rotational flow, generated near the cuff exit.

\subsection{Heat transfer characteristic from cuff surface}

Complicated flow, including rotational flow, through the cuff facilitates heat transfer to a large extent within the cuff surface. The purpose of the cuff is to cool the biotissue in the pharynx division. Therefore, it is important to understand the heat transfer characteristics between the cooling water and cuff wall surface through which the water flows. Next, the cooling water inflow (V) and amount of heat transfer (Q) calculated from the temperature gradient $(\triangle \mathrm{T})$ were examined. The relationship between the cooling water inflow and

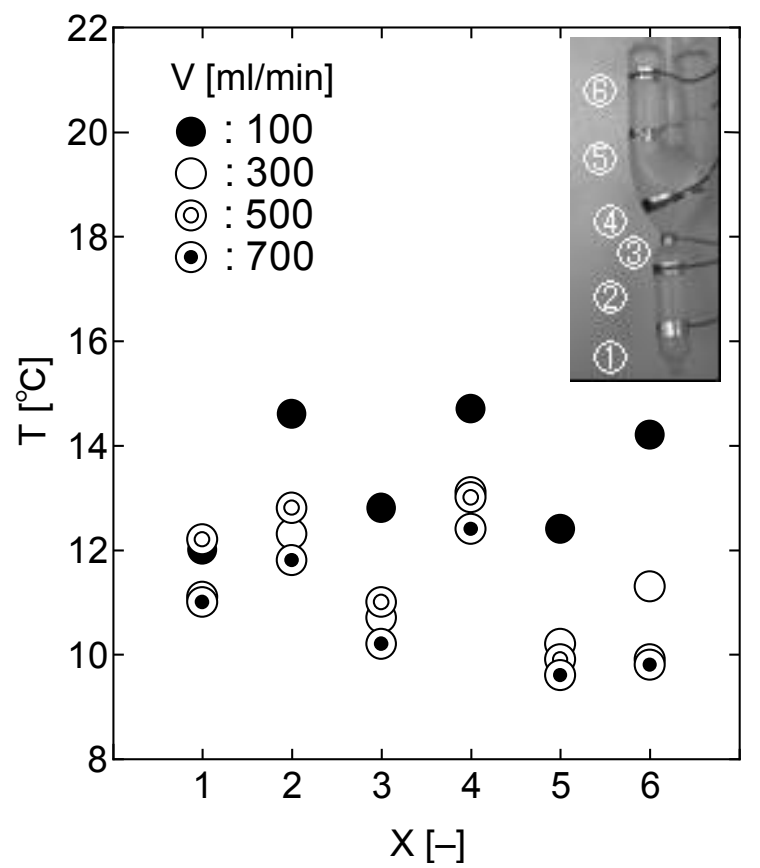

Figure 5 Temperature distribution in the cuff within the phantom 
amount of heat transfer and cooling efficiency $(\eta)$ of the cuff is shown in Figure 6 . The cooling efficiency is calculated with the following equations.

$$
\eta=\frac{T_{\text {out }}-T_{\text {in }}}{T_{\text {Pha }}-T_{\text {in }}} \times 100
$$

Where, $\mathrm{T}_{\text {in }}, \mathrm{T}_{\text {out }}$, and $\mathrm{T}_{\mathrm{Pha}}$ are the cuff inlet temperature, outlet temperature, and phantom temperature, respectively. The phantom temperature and inflow temperature were fixed at $\mathrm{T}_{\text {Pha }}=37^{\circ} \mathrm{C}$ and $\mathrm{T}_{\text {in }}=5^{\circ} \mathrm{C}$. Since the sensible heat traffic volume increases, as the inflow increases, there is a synchronous increase in the amount of heat transfer. In addition, the rapid flow, including the rotational flow, generated in the cuff due to the increased inflow is considered to be a factor inducing an increase in the amount of heat transfer. Moreover, it has been proven that the cooling efficiency exponentially decreases with an increase in the inflow. This is because the increasing temperature gradient $(\Delta \mathrm{T})$ of the inflow decreases with increase in the inflow. In particular, the cooling efficiency stabilizes at approximately $6 \%$ at an inflow of over $750 \mathrm{ml} / \mathrm{min}$.

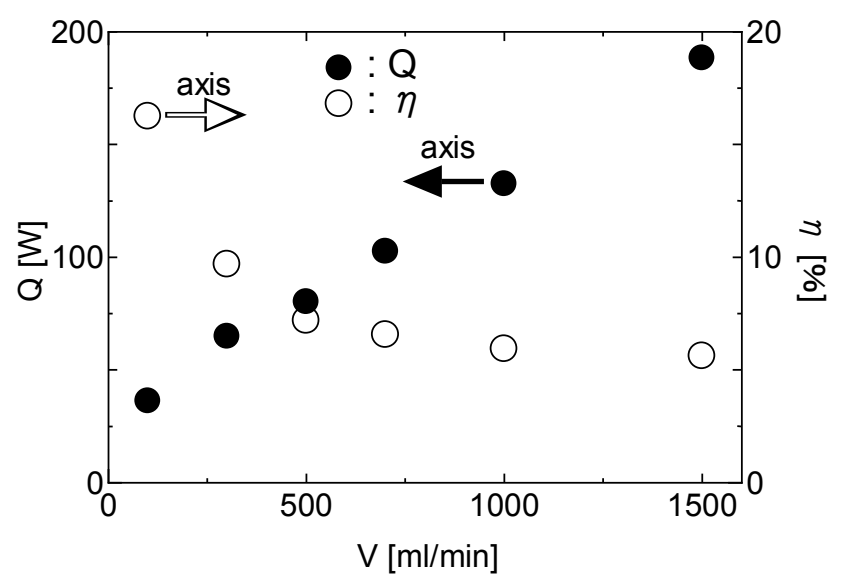

Figure 6 Relation between heat quantity and cooling efficiency

\subsection{Flow characteristics in cuff}

Numerical results of temperature distribution (left side) and cuff flow pattern (right side) are shown in Figure 7. (a) and (b), respectively, in the case of cooling water inflows of $500 \mathrm{ml} / \mathrm{min}$ and $150 \mathrm{ml} / \mathrm{min}$. The inflow temperature was $\mathrm{T}_{\text {in }}=5^{\circ} \mathrm{C}$ in both cases. The temperature conditions of the cuff surface during the analysis have been set as follows. On the basis of the relationship between heat quantity and cooling efficiency in Figure 6, in the boundary condition, the heat flux (q), which was divided by the cuff surface area ( $\mathrm{S}=$ $0.021 \mathrm{~mm})$, was calculated in terms of the amount of heat transferred (Q). The heat flux (q) at $\mathrm{V}=500 \mathrm{ml} / \mathrm{min}$ and $150 \mathrm{ml} / \mathrm{min}$ were $3810 \mathrm{~W} / \mathrm{m}^{2}$ and $2068 \mathrm{~W} / \mathrm{m}^{2}$, respectively. The temperature distribution in the cross section of the cuff is shown in Figure 7 . In the right figure, the three-dimensional locus of the representative particles is shown in order to demonstrate the flow characteristics in the cuff in terms of the fluid particles. On the basis of the temperature distribution, we observe that the temperature at the tip and in the central section of the cuff is low. In addition, the temperature at the point whereof the fluid pools, i.e., in the area of the rotational flow, is also low. These results are in agreement with the measurement results in Figure 5. Furthermore, the temperature in the cuff in general decreases with the increase in the inflow, and there is insufficient heat exchange. Moreover, as seen in the flow pattern in Figure 8, the fluid in the cuff exhibits a rotational flow in the cuff tip, and it turns at the upper part of the cuff. It was confirmed that there is paired 
rotational flow at the cuff center for right and left. These flow patterns are largely affected by the change in the inflow. As shown in Figure 7, it is understood that the flow at the cuff central part is runoff without reaching the cuff surface by the rotational flow. This means that the cooling water which has been flowing from below the cuff did not contribute to the heat transfer with the cuff surface. Moreover, it is proved that the central part of the rotational flow is the stagnation point. From these points of view, it seems to be possible that the thermal efficiency increases by suppressing the rotational flow in the cuff. The images in Figure 8 (a) and (b) show the streamlined flow in each part of the cuff. Figure 8 (a) shows the central portion of the cuff and (b) shows the cuff tip. The total inflow is 300 $\mathrm{ml} / \mathrm{min}$. Figure 8 (a) shows the paired rotational flow for the right and left after the fluid pooled in the cuff bifurcation and after it was distributed in the arms. After the intense rotational flow in the cuff tip, the fluid turns centrally and flows in a rectilinear manner (Figure 8 (b)). The flow characteristics are in accordance with the numerical result shown in Figure 7.

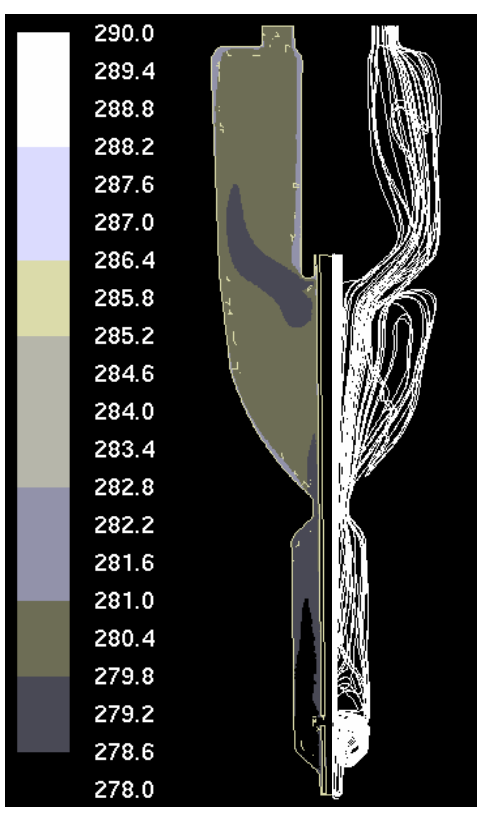

(a) $\mathrm{V}=500 \mathrm{ml} / \mathrm{min}$

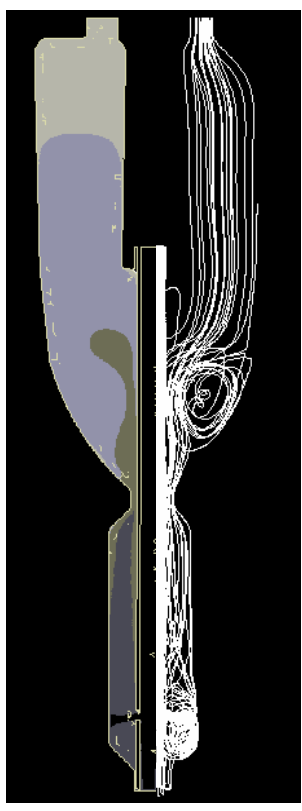

(b) $\mathrm{V}=150 \mathrm{ml} / \mathrm{min}$

Figure 7 Numerical results of temperature distribution and flow patterns

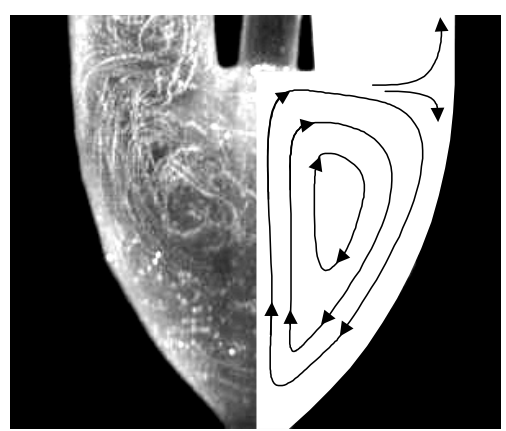

(a) Middle part

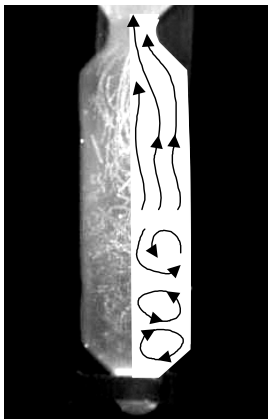

(b) Apical part

Figure 8 Results of the visualization experiment 


\section{Conclusion}

The use of the pharyngeal cooling cuff for the treatment of brain hypothermia was experimentally and numerically analyzed, and this analysis led to the following conclusions.

(1) It was possible to clarify the heat- transfer characteristics of the pharyngeal cooling cuff experimentally and numeric-analytic. Furthermore, the results of this simulation result agreed with the experimental results.

(2) The cooling efficiency and amount of heat transfer in the cuff are dependent on the inflow. However, it is considered that heat transport efficiency and local heat exchange lower by the rotational flow in the cuff. The cooling efficiency stabilizes at $6 \%$, when the inflow is greater than $750 \mathrm{ml} / \mathrm{min}$. This is a very low value, and further improvements in the cuff are necessary. Currently we are performing both experimental and computational analysis on a cuff design which suppresses the rotational flow structure.

\section{Acknowledgements}

This study was financially supported by Health Labour Sciences Research Grant, Japan. And the simulation analysis of the cuff was carried out using a High Performance computer at the Hokkaido University Information Initiative Center.

\section{References}

(1) National Institute of Population and Social Security Research, Population Statistics of Japan, (2008). (in Japanese)

(2) N. Hayashi and Dalton W. Dietrich, Brain Hypothermia Treatment, Springer-Verlag, (2000).

(3) N. Hayashi, N. R. Bullock, et al., Hypothermia for Acute Brain Damage, Springer-Verlag, (2004).

(4) The hypothermia after cardiac arrest study group, Mild therapeutic hypothermia to improve the neurologic outcome after cardiac arrest. N. Engl. J. Med. Vol. 346, (2002), pp.549-556.

(5) S. A. Bernard, T. W. Gray and M.D. Buist, et al., Treatment of Comatose Survivors of Out-of-Hospital Cardiac Arrest with Induced Hypothermia , N. Engl. J. Med. Vol. 346, (2002), pp.557-563.

(6) W. Rasch and M. Cabanac, Selective brain cooling is affected by wearing headgear during exercise, J. of Appl. Phys., Vol. 74, Issue 3, (1993), pp.1229-1233.

(7) S. Higano, Y. Takeda, K. Takata and K. Morita, Nasopharyngeal cooling selectively and rapidly decreases brain temperature and attenuates neuronal damage, even if initiated at the onset of cardiopulmonary resuscitation in rats, Crit. Care Med., Vol. 31, (2003), pp. 2502-2508.

(8) K. Takata, Y. Takeda and T. Sato, et al., Effects of hypothermia for a short period on histlogic outcome and extracellular glutamate concentration during and after cardiac arrest in rats, Crit. care Med., Vol. 33, (2005), pp.1340-1345.

(9) Y. Takeda, The new brain cryogenic technique : Pharyngeal cooling and trend of Neurotransmitter, 11th Proc. of Japanese Association of Brain Hypothermia, (2008)). (in Japanese)

(10) Thermophysical Properties Handbook, Japan Society of Thermophysical Properties, Yokendo LTD., (1990). (in Japanese) 